\title{
BODY PART SEGMENTATION OF NOISY HUMAN SILHOUETTE IMAGES
}

\author{
Mark Barnard, Matti Matilainen and Janne Heikkilä \\ Machine Vision Group \\ Department of Electrical and Information Engineering \\ University of Oulu, Finland
}

\begin{abstract}
In this paper we propose a solution to the problem of body part segmentation in noisy silhouette images. In developing this solution we revisit the issue of insufficient labeled training data, by investigating how synthetically generated data can be used to train general statistical models for shape classification. In our proposed solution we produce sequences of synthetically generated images, using three dimensional rendering and motion capture information. Each image in these sequences is labeled automatically as it is generated and this labeling is based on the hand labeling of a single initial image. We use shape context features and Hidden Markov Models trained based on this labeled synthetic data. This model is then used to segment silhouettes into four body parts; arms, legs, body and head. Importantly, in all the experiments we conducted the same model is employed with no modification of any parameters after initial training.
\end{abstract}

Index Terms - body part recognition, silhouette segmentation, shape context features

\section{INTRODUCTION}

Identification of body parts is an important first step for many tasks, such as action recognition, tracking and pose estimation. Methods for identifying body parts fall into two broad categories: model based and learning methods. Model based approaches are top-down methods where prior knowledge of the shape of the human body in various poses and the location of the limbs in these poses is used for part identification. These models are often used for tracking the location of the limbs in video sequences [3] [4]. Mittal et al [5] present an algorithm for body part segmentation from silhouette images. Their approach divides the body based on negative minimum curvature. The authors report that their algorithm performed poorly when the body parts, particularly the arms are held close to the body. Much of the work in this area has concentrated on reconstructing a full three dimensional (3-D) pose from the silhouette data. Li et al[6] use general geometric transform in their approach where a visual pattern is represented with certain invariance through this transform before learning is applied. In Agarwal and Triggs[7] present a learning based approach in which a set of 54 three dimensional limb angles are recovered from a silhouette image by regressing against the shape context features for the silhouette image. Mori and Malik[8] use shape context features to match test silhouettes to a set of exemplars.

Our goal in this work is to demonstrate that using artificially generated training data we can produce a segmentation of the human outline taken from noisy real silhouette images. This segmentation should be able to perform well despite noise in the body silhouette outline. To demonstrate this we first train models on synthetic data with labeled body part segments viewed in a range of different body configurations and from a range of different viewing angles. Using a labeled validation set also comprised of synthetic data sequences we are able to set all the hyper-parameters of the model. We show that a model trained on synthetic data can perform well regardless of changes in viewing angle, occlusions and noise in the image. We also show that the same model, with no changes to any parameters, can give good results on data collected from different sources in different environments with different subjects.

Synthetic data has been used effectively in other fields where the amount of training data is limited. Varga and Bunke [1] proposed a perturbation model for creating synthetic training data for handwritten text recognition. While, Heisele and Blanz [2] use morphable models to create more training data from a face database that had only a small number of images per person.

In Section 2 we give a background to shape context features and propose a modification to improve performance in noisy environments. We use a Hidden Markov Model (HMM) to model the silhouette outline as a sequence of body parts. A general model is trained using shape context features extracted from the labeled synthetic data. Details of this approach are given in Section 3. The performance of this model is tested in a series of experiments described in Section 4.

\section{SHAPE CONTEXT FEATURES}

In order to capture the shape of the silhouette outline we use shape context features introduced by [9]. These are a generic set of features used to the describe the shape of a silhouette outline. Originally used in shape matching and defining the aligning transformation between two objects. Shape context features have been used for 3-D pose recovery from 2-D images $[8,7]$ and Poppe and Poel [10] compared Shape Context Features to Fourier descriptors and $\mathrm{Hu}$ Moments. They recovered human pose from large database of human silhouettes under different view angles, body dimensions and noise. The locality of Shape Context features was found to overcome the problem of artifacts or noise in one area of the silhouette affecting the entire descriptor. This property of locality also aids in the recognition of partially occluded shapes.

To create shape context features a silhouette edge is sampled at regular intervals. For each sampled edge point the distance and direction to all other edge points that fall under the maximum distance are calculated. The distances and directions are stored in a log-polar histogram. In the original description of shape context features the spacing of the radial bins was logarithmic. This was to increase the locality of the feature, however we found that in the presence of noisy silhouettes logarithmic spacing places too much emphasis 


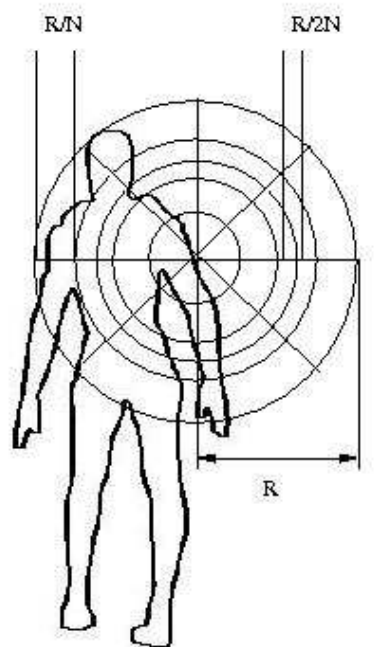

Fig. 1. A shape context descriptor showing our proposed use of weighted bins.

on local pixels thus may cause recognition errors. We, therefore, propose a weighting on the radial bin size as shown in Figure 1. We define the overall radius of the shape context descriptor as $R$ with the width of each radial bin being $d(r)$ and the distance from the centre of the descriptor as $r$. Instead of the original logarithmic spacing we use a weighted spacing of the radial bins according to,

$$
d(r)= \begin{cases}\frac{R}{2 N} & \text { if } \frac{R}{3}<r<\frac{2 R}{3} \\ \frac{R}{N} & \text { else }\end{cases}
$$

where $N$ is the number of radial bins with equal spacing. This places more emphasis on the pixels in the middle distance whilst still maintaining a degree of locality in the descriptor.

These shape context features are extracted from a whole body silhouette. The feature vectors corresponding to each body part are then selected based on the labeling of the synthetic model.

\section{OUR APPROACH}

The synthetic data used for our experiments was created through motion capture. In this process markers attached to the joints of a person are tracked in three dimensions using data recorded from multiple cameras. 3-D rendering software is then used to create human figures and the motion capture data is used to animate these figures. One of the main advantages of generating synthetic training data is that the skin used in the 3-D rendering of the sequences can be labeled according to body parts. This labeling needs only to be performed manually once, then this pre-labeled skin can be applied to different skeletons. These skeletons, with the attached skin, is then animated using the motion capture data. So instead of, tediously, manually labeling each frame, we can label a single frame and this labeling is carried through for all subsequent frames in the training set as the figure adopts different poses.

Five subjects were used to create the synthetic training set: 3 male and 2 female. Each subject was recorded performing two action sequences: SEQ1 walk across the room, sit on a chair, walk back across the room, SEQ2 walk across the room, fall over and then get

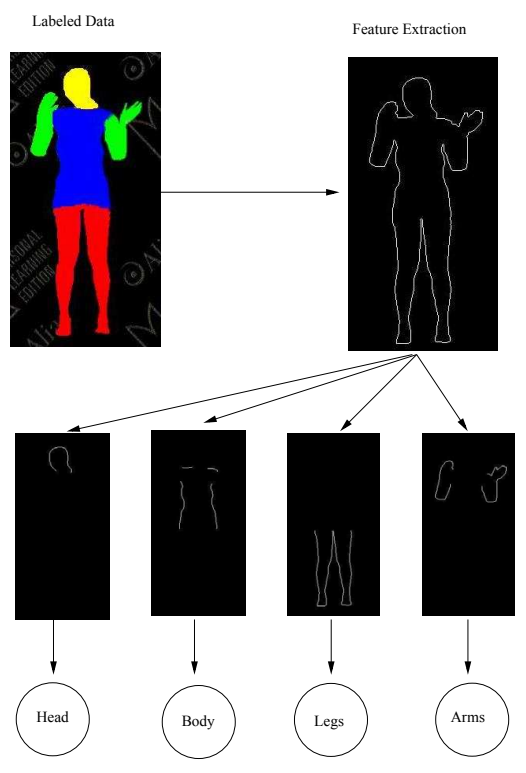

Fig. 2. The method for selecting GMM training data based on automatic body shape labeling

up, walk back across the room. Each sequence was then rendered from six different angles: from the front and offset by -20 and +20 degrees, from the side, again offset by -20 and +20 degrees.

The labelled silhouettes created by the 3 -D rendering process are used to generate sets of labeled shape context features, described in Section 2, to train a Gaussian Mixture Model (GMMs) for each body part. The selection of training data is shown in Figure 2, the colour labeling of the rendered figure is used to segmented the features based on body parts. These GMMs form the states of a HMM [11]. We can consider each silhouette outline as a sequence of shapes corresponding to body parts. Using an HMM we can constrain the shape recognition by taking into account the transitions between different body parts using Viterbi decoding [12]. Instead of determining these transitions from a priori domain knowledge, that may be flawed or not related directly to the data set we are dealing with, we determine the transitions from the training data itself using the labeled synthetic data, as shown in Figure 3. This gives us a distribution of the transitions probabilities between body parts actually within the data. So we train a four state fully-connected or ergodic HMM using the labeled synthetic data. The model contains one state for each body part, in our case Head, Body, Arms and Legs.

\section{EXPERIMENTS}

In our experiments we test the performance of the proposed body part recognition method on sequences containing people in an number of normal human poses, such as standing and walking. The tested material consists of sequences of noisy, poorly segmented silhouettes taken from a number of different sources. These sequences feature different subjects recorded from a variety of different viewing angles and also in the presence of occlusions. In order to create the silhouettes we used simple background subtraction. While there are more sophisticated methods available they often require tuning for a particular data set and we felt that the simplest method would produce a good baseline for tesing our approach.

The aim is to classify each pixel on the silhouette outline into 


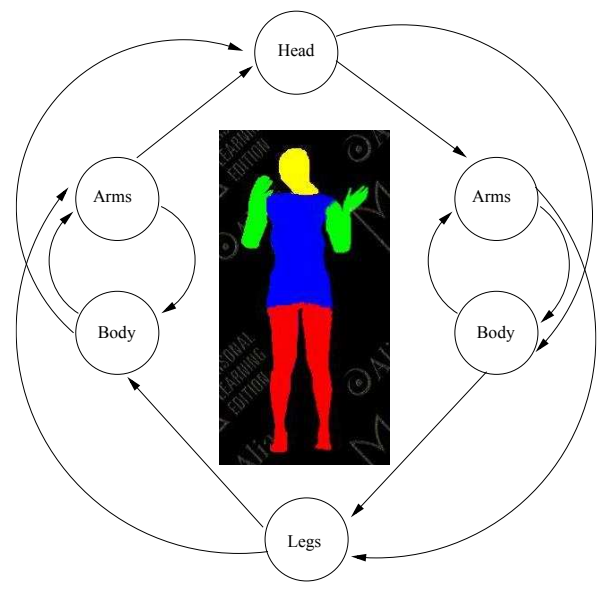

Fig. 3. Transition between body parts used to constrain body part shape recognition.

one of four body part classes, Head, Body, Arms, Legs. In order to cope with uncertainty in the recognition process we use a ratio of the likelihood between the most likely class and the second most likely class for each pixel on the silhouette outline. Any pixel where the likelihood falls below a certain threshold is discarded as the uncertainty on the pixel is considered too high.

The results of performing body part segmentation on silhouettes in various poses and from different viewing angles is shown in Figure 4. The images are composed of three sections the first is the original frame from the video sequence, the second is the silhouette outline obtained from using simple background subtraction and the third is the silhouette outline segmented into body parts. These results indicate that our method is able to accurately segment the silhoutte into body parts regardless of changes in the horizontal viewing angle.

A significant problem in dealing with silhouette data is that it can be very noisy. This noise is in the form of shadows, changes in lighting conditions or the background matching the foreground we wish to segment. This noise can cause dramatic changes in the shape of the silhouette and so seriously degrade the performance of any shape recognition method. In Figure 5 we show the performance of our model in noisy conditions. In the first image it can be seen that even though the outline of the arms has been broken, it is still clearly recognised as an arm. In the second and third image although part of the limb, in the second a leg and the third an arm, have been badly segmented this does not affect the overall recognition of the limb. Indeed in the fourth image one entire leg is lost due to noise and yet the remaining leg is still recognised correctly. The two remaining images shows noise caused by shadows from the door giving very poor segmentation of the legs.

Another source of error in silhouette segmentation is occlusions. This is particularly true in the case of surveillance and monitoring applications where a subject may be entering or leaving a room. Figure 6 shows frames taken from a sequence where the subject enters and leaves the frame a number of times. The first three frames are from a sequence taken at five frame intervals of the subject exiting the frame. It can be seen that a relatively good limb segmentation is maintained throughout the sequence. Although as the amount of occlusion increases the number of rejected pixels increases, showing an increase in the uncertainty about the overall shape of the silhouette outline. The last two images show horizontal occlusions caused by

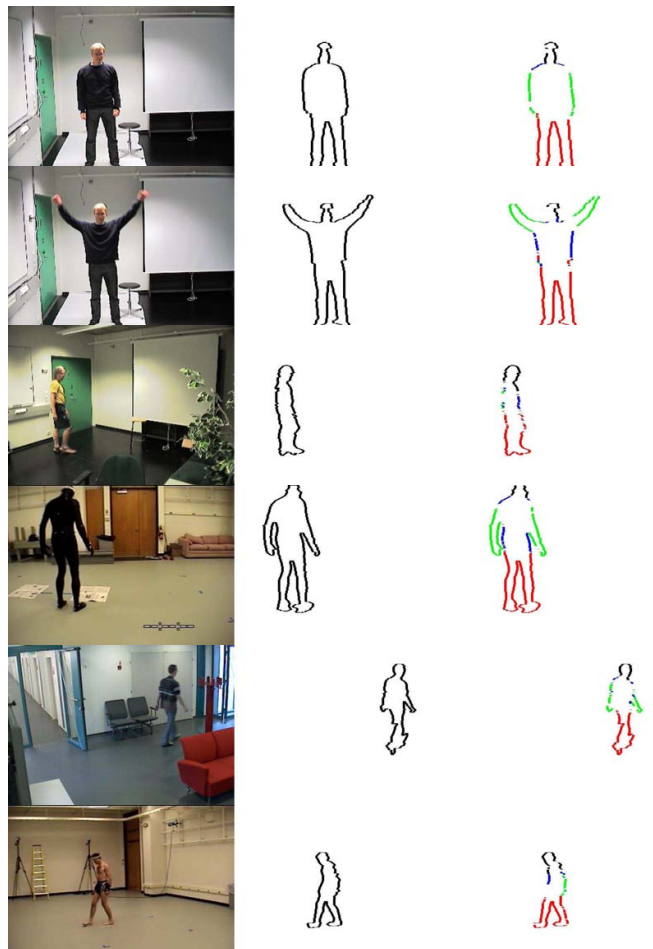

Fig. 4. Subjects in different poses from various angles, demonstrating the robustness of the body part segmentation to viewing angle.
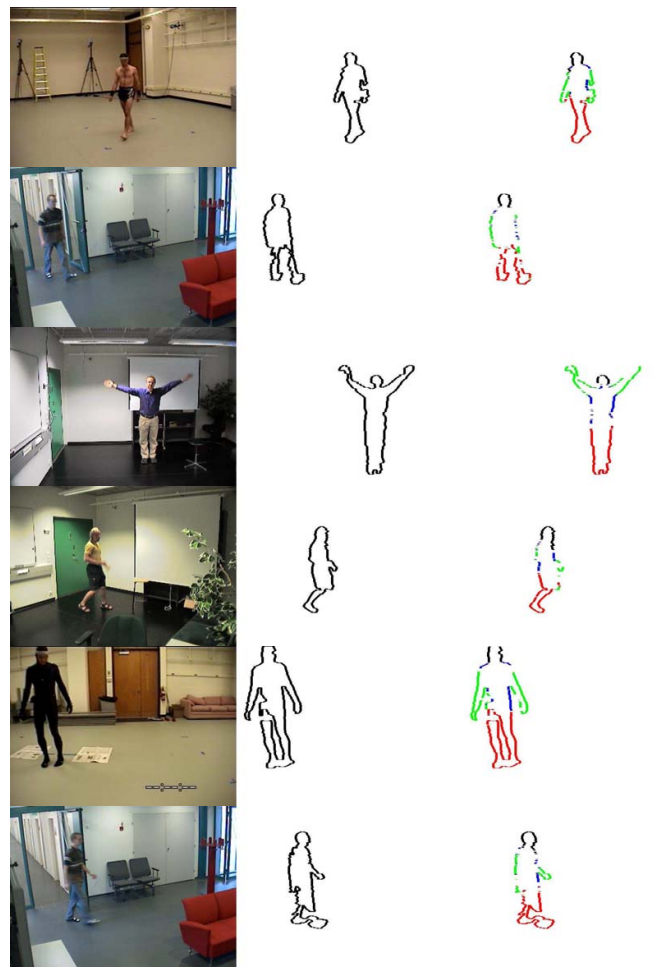<smiles>O=[V]</smiles>

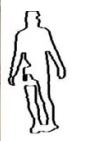<smiles>c1ccccc1</smiles>
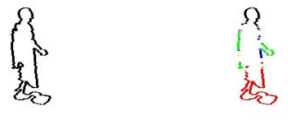

Fig. 5. A selection of noisy, badly segmented silhouettes, showing our methods robustness to noisy silhouette outlines. 


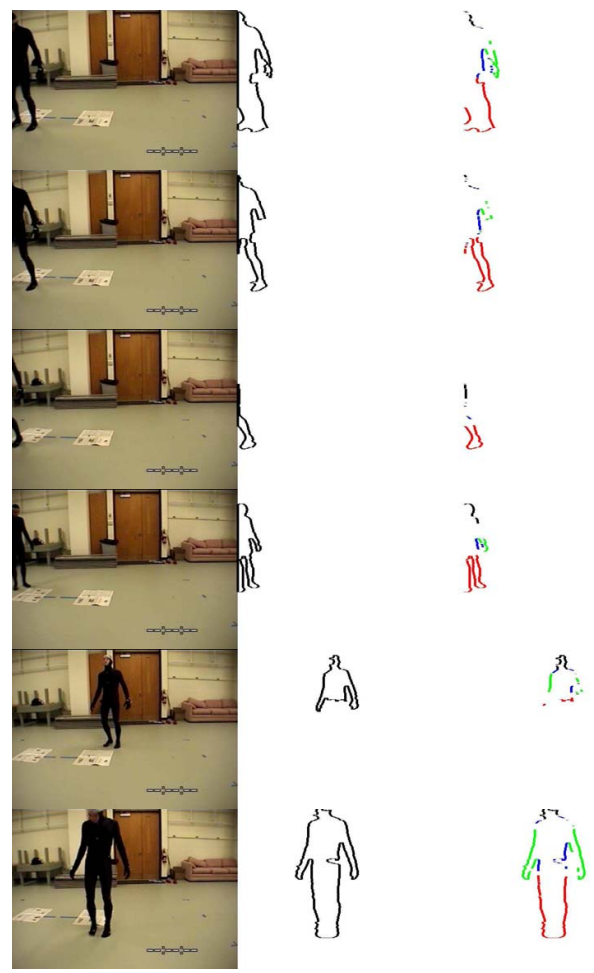

Fig. 6. A subject under different types of occlusion. These results show the performance of our algorithm under various degrees of occlusion.

noise. In the first the entire lower portion of the silhouette is missing, while there are some errors the arms and head of the subject are still being clearly recognised. In the second most of the head is missing yet this has almost no effect on the rest of the segmentation.

\section{CONCLUSIONS}

We have presented here a system for producing large amounts of labeled training data. This data is based on sequences of synthetic images rendered from motion capture data. This synthetic data can be produced from a variety of viewing angles with any desired labeling. This overcomes one of the fundamental problems in statistical video and image analysis, this being the lack of labeled training data. We demonstrate that a model produced using this synthetic data can be applied to the segmentation of real body silhouettes and is able to achieve good results regardless of viewing angle, noise or occlusions. The general nature of this model was demonstrated by the fact that even though the test set consisted of videos produced in a different environment, with different subjects, resolution and frame rate to the training data, none of the model parameters were adjusted after the initial training and validation.

The system we have introduced here has, we believe, the potential to be used in many different applications. For example the position of body parts could be tracked through a sequence of images and then the trajectories of the body parts could be used to recognise more complex events. There is also the potential for using models such as this for human vs non-human silhouette identification.

\section{REFERENCES}

[1] T. Varga and H. Bunke, "Generation of synthetic training data for an hmm-based handwriting recognition system.," in In proceedings International Conference on Document Analysis and Recognition, 2003.

[2] B. Heisele and V. Blanz, "Morphable models for training a component-based face recognition system," in In Proceedings Computer Vision and Patern Recognition, 2004.

[3] C. Bregler and J. Malik, "Tracking people with twists and exponential maps," in CVPR '98: Proceedings of the IEEE Computer Society Conference on Computer Vision and Pattern Recognition, Washington, DC, USA, 1998, p. 8, IEEE Computer Society.

[4] H. Sidenbladh, M. J. Black, and L. Sigal, "Implicit probabilistic models of human motion for synthesis and tracking," in In proceedings European Conference on Computer Vision, 2002.

[5] A. Mittal, Liang Zhao, and L.S. Davis, "Human body pose estimation using silhouette shape analysis," in In proceedings. IEEE Conference on Advanced Video and Signal Based Surveillance, July 2003, pp. 263-270.

[6] Jian Li, Shaohua Kevin Zhou, and Rama Chellappa, "Appearance modeling under geometric context," in ICCV '05: Proceedings of the Tenth IEEE International Conference on Computer Vision, Washington, DC, USA, 2005, pp. 1252-1259, IEEE Computer Society.

[7] A. Agarwal and B. Triggs, "Recovering 3d human pose from monocular images.", IEEE Transactions on Pattern Analysis and Machine Intelligence, vol. 28, no. 7, pp. 1052-1062, 2006.

[8] G. Mori and J. Malik, "Recovering 3d human body configurations using shape contexts.," IEEE Transactions on Pattern Analysis and Machine Intelligence, vol. 28, no. 7, pp. 10521062, July 2006

[9] S. Belongie, J. Malik, and J. Puzicha, "Shape matching and object recognition using shape contexts.," IEEE Transactions on Pattern Analysis and Machine Intelligence, vol. 24, no. 4, pp. 509-522, April 2002.

[10] R. Poppe and M. Poel, "Comparison of silhouette shape descriptors for example-based human pose recovery.," in Proceedings of the 7th International Conference on Automatic Face and Gesture Recognition., 2006.

[11] Lawrence Rabiner and Biing-Hwang Juang, Fundamentals of Speech Recognition, PTR Prentice Hall, 1993.

[12] A. J. Viterbi, "Error bounds for convolutional codes and an asymptotically optimal decoding algorithm.," IEEE Transactions Information Theory, vol. IT-13, pp. 260-269, 1967. 